\title{
Transcriptional Control in Keratinocytes and Fibroblasts Using Synthetic Ligands
}

Rachel A. Freiberg, ${ }^{*}$ Steffan N. Ho, ${ }^{\ddagger}$ and Paul A. Khavari ${ }^{\star}$

Dermatology Service, VA Palo Alto Health Care System, Palo Alto, California 94304; and the Departments of*Dermatology, ${ }^{\ddagger}$ Developmental Biology, and the Howard Hughes Medical Institute, Stanford University, Stanford, California 94305

\begin{abstract}
The skin is an attractive tissue for regulated target gene expression by virtue of its accessibility to topical regulating stimuli. We have used synthetic ligand-driven intracellular oligomerization to accomplish specific target gene regulation in human skin keratinocytes and fibroblasts. GAL4 DNA binding domains and VP16 transactivation domains, each linked to the FK506 binding protein, were expressed in normal human skin keratinocytes and fibroblasts. These hybrid proteins underwent heterodimerization via the novel intracellular dimerizing agent FK1012 to generate a heterodimeric activator of target gene expression in vitro. Dimeric FK1012, but not monomeric FK506M induced target gene expression in a dose-dependent fashion. FK1012 exerted no detectable nonspecific effects on expression of cutaneous genes and did not alter cellular proliferation kinetics. Controlled oligomerization of hybrid transcription activators offers a potential approach to target gene regulation in cells of normal human skin. (J. Clin. Invest. 1997. 99:2610-2615.) Key words: gene regulation • skin • transcriptional control • keratinocytes $\bullet$ fibroblasts
\end{abstract}

\section{Introduction}

The ability to control the magnitude of expression of specific target genes in intact cells and tissues holds the promise of performing complex genetic experiments with quantitative biochemical precision as well as for delivering dosed genetic therapies. One example of the importance of the magnitude of gene expression involves the relative expression of Bcl-2 and Bax in cell death where the level of expression of each protein determines a critical ratio of $\mathrm{Bcl}-2$ to Bax protein that is decisive in determining if a cell will die or live in response to certain stimuli (1). The skin is an attractive tissue for exogenously controlled experimental and therapeutic gene regulation in vivo because of its accessibility and proven ability to deliver polypeptides to the systemic circulation (2). Currently available approaches to accomplish precise cutaneous gene regulation have major limitations. Topical glucocorticoids and retinoids have been therapeutic mainstays for many years but these steroid receptor family binding molecules produce significant side effects, alter expression of a wide range of genes and display consistent background expression from responsive promoters (3). Other widely used approaches have major draw-

Address correspondence to Paul A. Khavari, Chief, Dermatology Service [123], VA Palo Alto Health Care System, 3801 Miranda Avenue, Palo Alto, CA 94304. Phone: 415-852-3494; FAX: 415-723-8762; E-mail: khavari@cmgm.stanford.edu

Received for publication 15 May 1996 and accepted in revised form 27 February 1997.

The Journal of Clinical Investigation

Volume 99, Number 11, June 1997, 2610-2615 backs as well. Those among the best known involve the mouse metallothionein promoter (4) and the tetracycline (Tet) repressor (5). In the case of the metallothionein promoter, administration of metals is necessary to activate gene expression, itself associated with certain toxicities and with activation of other subsets of genes. In the case of the Tet system, the relatively broad spectrum antibiotic action and effects on inflammation of tetracycline along with the topical photosensitizing potential of this agent limit its usefulness in the skin. Utilization of the epidermis for experimental and therapeutic target gene regulation has awaited development of new approaches.

Targeted intracellular oligomerization of engineered transcription factors offers a promising avenue to accomplish regulated target gene transcription in a range of tissues. Oligomerization of biomolecules to generate a biologic response is a recurrent theme in biology in a wide variety of processes. Two examples include cell surface receptor cross-linking (6) and transcription activator dimerization (7). In the case of cell surface receptor cross-linking, among the best investigated system is the mammalian T lymphocyte surface antigen receptor (8). A well-studied example in gene transcription involves AP-1 where jun and fos family members heterodimerize to specifically alter gene expression (9). While the use of specific antibodies has allowed targeted cross-linking of extracellular or cell surface proteins (10), a general method to oligomerize molecules inside living cells with precise specificity-a key to recapitulating and regulating major intracellular processes such as signal transduction and gene expression-has, until recently, not been available. A novel approach to accomplishing this task has emerged in the form of the intracellular dimerizing agent FK1012, constructed as a dimer of the immunosuppressant macrolide FK506 (11). Dimeric FK506 (FK1012), in contrast to FK506 monomer, exerts no apparent immunosuppressive effects (11). FK1012 is capable of cross-linking proteins engineered to contain the hydrophobic pocket of its intracellular ligand, FK506 binding protein (FKBP), ${ }^{1}$ at high efficiency. FK1012 has been used to trigger antigen receptor signal transduction pathways in transformed lymphoid cells (11). Interestingly, topically applied FK506 based compounds readily penetrate into the epidermis by virtue of their hydrophobic nature and exert effects on hair follicle growth kinetics (12) and inflammation (13). Here we have applied this approach to take the first step to developing regulated cutaneous gene expression by controlling the magnitude of gene expression in normal diploid human skin keratinocytes and fibroblasts in vitro utilizing a novel FK1012-driven transcription system.

\section{Methods}

Cell harvesting and tissue culture. Primary human keratinocytes (14) and fibroblasts (15) were isolated from neonatal foreskin and grown

1. Abbreviations used in this paper: CAT, chloramphenicol acetyltransferase; CMV, cytomegalo virus; fkBP, fk506-binding protein; $\mathrm{k} 5$, keratin 5. 
as previously described. Keratinocytes were cultured in growth media consisting of $50 \%$ serum-free medium plus supplements (SFM; Gibco, Grand Island, NY), 50\% Medium154 plus supplements (Cascade Biologics, Portland, OR), amphotericin $0.05 \mathrm{ng} / \mathrm{ml}$, and gentamycin $0.05 \mu \mathrm{g} / \mathrm{ml}$. Fibroblasts were cultured in growth media consisting of Iscove's Modified Dulbecco's Medium (Gibco), 10\% bovine calf serum (Hyclone Laboratories, Logan, UT), penicillin-streptomycin, Hepes, L-glutamine. Cells were incubated at $37^{\circ} \mathrm{C}$ in $5 \% \mathrm{CO}_{2}$ air atmosphere. The effects of FK1012 on proliferation were determined with the cells grown in triplicate in the presence or absence of $100 \mathrm{nM}$ FK1012. The population of cells was determined by counting at triplicate cultures at serial timepoints in keratinocytes fibroblasts growing from sparse density to confluence.

Gene transfection. Primary human keratinocytes were cultured in 35 -mm plates to $\sim 70-80 \%$ confluency then were transfected by lipofection (16). In lipofection, $283 \mu \mathrm{l}$ serum-free medium without antibiotics was mixed with $17 \mu \mathrm{l}$ of $1 \mathrm{mg} / \mathrm{ml} \mathrm{lipofectin} \mathrm{(Gibco).} \mathrm{After} 10$ min of incubation at room temperature the mixture was added to the cells and incubated at $37^{\circ} \mathrm{C}$ for $3-6 \mathrm{~h}$. At the end of the incubation period, the medium was replaced with complete growth medium and the cells were incubated overnight at $37^{\circ} \mathrm{C}$. Cell extracts were prepared and analyzed for luciferase and chloramphenicol acetyl-transferase (CAT) activity. Primary human fibroblasts were cultured in 35 -mm plates to $\sim 70-80 \%$ confluence then were transfected by modified polybrene shock (16). For polybrene transfection $760 \mu \mathrm{l}$ of growth media was mixed with the plasmid to be transfected and are vortexed vigorously. $3.8 \mu \mathrm{l}$ of $1 \mathrm{mg} / \mathrm{ml}$ hexadimetherine bromide (Aldrich Chemical Co., Milwaukee, WI) in HBSS was added and again vortexed. This mixture was overlaid on the cells for $6 \mathrm{~h}$. A $28 \%$ DMSO (Sigma Chemical Co., St. Louis, MO) in growth media mix was applied to the cells after the media has been aspirated. The cells were incubated for $90 \mathrm{~s}$ before the DMSO was aspirated and replaced with PBS containing $10 \%$ bovine calf serum. The plates were rinsed twice and fresh growth media added, then cells were incubated overnight at $37^{\circ} \mathrm{C}$. Cell extracts were prepared and analyzed for luciferase and CAT activity. The GAL4-FKBP and FKBP-VP16 expression constructs in PBJ5 and the reporter plasmid with GAL4 sites driving luciferase were constructed previously (17). For glucocorticoid-driven transcription, the GRE-luciferase reporter plasmid (generous gift of K. Yammamoto) was used and transcription activated with dexamethasone (Sigma Chemical Co.). All transfections were internally controlled with either cytomegalo virus (CMV) promoter-driven luciferase or CAT depending on the test reporter gene used and data normalized for transfection efficiency.

Analysis of FK1012 effects on keratinocyte and fibroblast gene expression. cDNA from keratinocytes and fibroblasts treated either with or without $100 \mathrm{nM}$ FK1012 was prepared with reverse transcriptase (18), modified for 500 cells. 500 cells were isolated, washed, and suspended in PBS then added in $1 \mu l$ volume to $20 \mu l$ reverse transcriptase lysis buffer as described (18). $2 \mu \mathrm{l}$ of resultant cDNA was analyzed with primers for the indicated genes. All samples were internally controlled for cDNA quality and reaction efficiency using primers for GAPDH.

\section{Results}

The magnitude of FK1012-driven gene expression is comparable to transfected endogenous keratinocyte and fibroblast promoters. The model system (17) tested relies on FK1012-driven heterodimerization of the inactive monomers GAL4-FKBP and FKBP-VP16 to reconstitute the potent transcriptional activator GAL4-VP16 (Fig. $1 a$ ). To determine the potency of our gene regulation system, we compared its activity to reporter gene activity driven by the human keratin 5 (K5) promoter (19), a constitutively active promoter in basal keratinocytes that has been extensively used to target gene expression to epidermis in transgenic mouse studies. As shown in Fig. $1 b$, FK1012-driven transcription is inducible from 13 to $470 \%$ that driven by a transfected K5 promoter-reporter construct, providing support for its ability to drive expression of target genes through the physiologic range in these cells. We confirmed that target gene expression could be activated by synthetic ligand in primary human fibroblasts as well; we compared its activity to the basal activity of the human stromelysin promoter (20) in primary fibroblasts. The magnitude of GAL4 site-responsive reporter gene activity driven by GAL4:FKBP and FKBP:VP16 in the absence and presence of $100 \mathrm{mM}$ FK1012 was compared with a transfected reporter gene driven by the native human stromelysin promoter (Fig. 1c). The magnitudes of target gene expression in this experiment lend support to the ability of FK1012 to drive expression of target genes from minimal levels up to physiologic ranges in these cells.

Concentration-dependent FK1012-driven gene expression. While a stoichiometric proportion of inactive monomers may form inactive homodimers in this model gene regulation system, those that heterodimerize would be predicted to form a functional activator molecule capable of more avid DNA binding than the inactive homodimers, by enhanced competition with nucleosomes for the specific promoter sites and activation of target gene transcription. After establishing that synthetic ligands could direct this system of gene expression in keratinocytes and fibroblasts, we wished to determine if this system would allow control of the magnitude of gene expression in a concentration-dependent manner. To do this, we tested if target gene expression could be regulated as a function of synthetic ligand concentration in primary human keratinocytes and fibroblasts. Primary human cells of both types transiently transfected in vitro and subjected to a range of FK1012 concentrations showed a dose-dependent activation of target gene expression (Fig. $2 a$ ). As can be seen, synthetic ligand concentrations in the $20 \mathrm{nM}$ range effectively activate target gene expression. The FK506M monomer, a molecule that binds at high affinity to FKBP but is incapable of acting as a dimerizing cross-linker, was entirely inactive in activating target gene expression. For comparison, an endogenous transcription control mechanism activated by cell permeable ligands, glucocorticoid receptor-driven transcription (21), was tested as well and demonstrated dose-dependent target gene activation (Fig. $2 b$ ). To determine the kinetics of synthetic ligand-driven target gene activation, a reporter gene induction time course was performed and demonstrated that reporter gene expression was activated within $60 \mathrm{~min}$ of synthetic ligand addition (Fig. 2c). These data demonstrate that this novel system of target gene expression in cells of the skin can be rapidly regulated in a synthetic ligand dose-dependent fashion.

FK1012 does not alter proliferation kinetics of primary human keratinocytes and fibroblasts. An evaluation of the toxicity of FK1012 in normal diploid human skin cells was necessary since initial studies utilizing synthetic ligands in the receptor, noted above, were performed in a transformed lymphoid line (11). We determined that FK1012 had no dramatic adverse effect on normal growth kinetics in vitro of primary human keratinocytes and fibroblasts, in cells growing form low density on tissue culture plates to near confluence (Fig. 3).

FK1012 does not dramatically alter mRNA expression of differentiation and tissue-specific genes in normal fibroblasts. To be useful in experimental and therapeutic cutaneous gene regulation, synthetic ligands, in contrast to steroid receptor 
ligands, should be highly specific for the targeted gene of interest and not adversely impact expression of other housekeeping or inducible genes. To screen for such nonspecific effects of the FK1012 synthetic ligand we examined mRNA levels of a number of constitutive and inducible genes in keratinocytes and fibroblasts in vitro in the presence or absence of FK1012. Using reverse transcriptase PCR internally controlled for GAPDH expression, we found no synthetic ligand effect on mRNA levels of all genes examined (Fig. 4). Expression of actively transcribed genes in keratinocytes, such as IL-1 $\alpha$, appear grossly uninhibited while genes normally silent in keratinocytes, such as pro 2 (I) collagen, do not appear activated by administration of FK1012. Furthermore, FK1012 did not appear to nonspecifically trigger keratinocyte differentiation in that it did not activate expression of K10, a marker of keratinocyte dif- ferentiation. Although K10 mRNA expression was undetectable in primary human keratinocytes treated with FK1012, it was readily seen in the well-characterized human HaCaT (22) cell line (Fig. $4 a$ ). In addition, FK1012 did not interfere with activation of collagenase or c-fos gene expression by PMA in fibroblasts and keratinocytes, respectively, suggesting a lack of nonspecific blocking effects on cellular gene induction processes (Fig. $4 b$ ).

\section{Discussion}

The magnitude of expression of individual genes within a living cell is often of central biologic importance and the ability to rapidly control this magnitude will have significant experimental and therapeutic applications. The ability to precisely

a

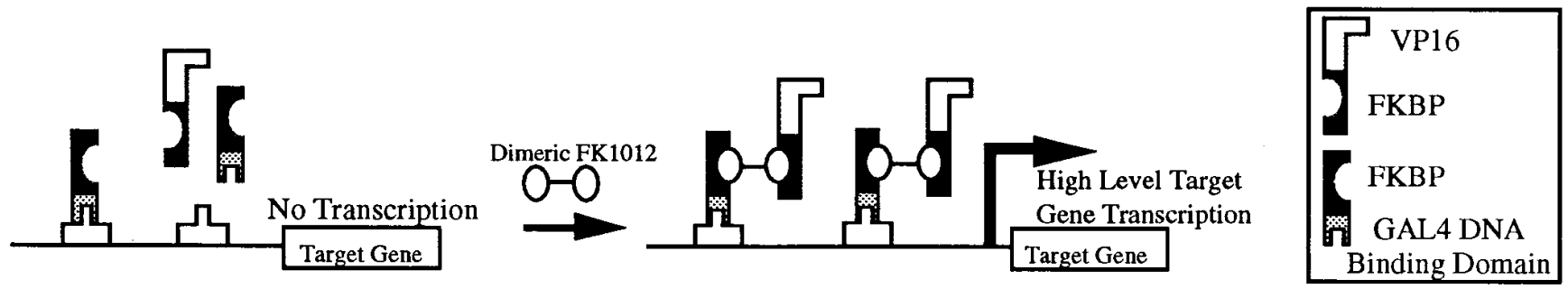

b

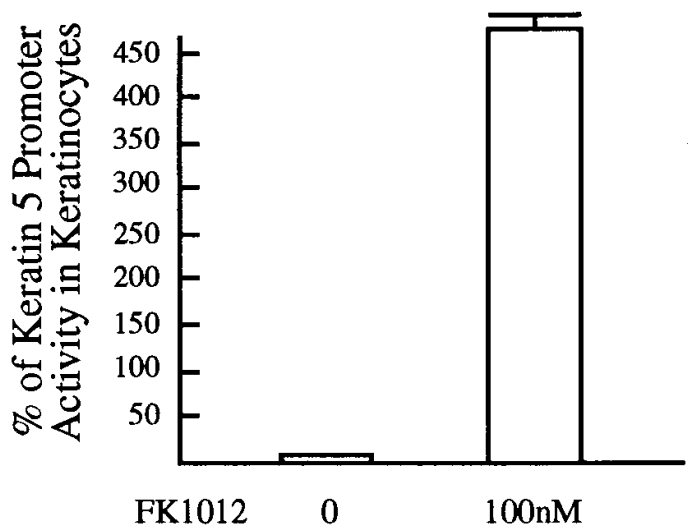

Figure 1. A synthetic ligand-driven promoter is active in primary keratinocytes and fibroblasts. (a) A flow diagram of the synthetic ligand driven system. GAL4FKBP $=$ DNA binding domain of the yeast transcription factor GAL4 (amino acids 1-147) fused to three tandem FK506 binding protein (FKBP) domains, FKBP-VP16 $=3$ tandem FKBPs fused to the activation domain of the herpes virus transactivating protein VP16 (amino acids 1-78) (17). Rectangular boxes in the promoter region diagrammed represent specific GAL4 DNA recognition sites. (b) The activity of the human keratin 5 promoter was compared with a synthetic ligand responsive GAL4 site-driven promoter in transiently transfected primary human keratinocytes in the absence or presence of $100 \mathrm{nM} \mathrm{FK1012.} \mathrm{Ke-}$ ratinocytes were transfected with synthetic ligand transcription expression and reporter plasmids or with the keratin 5 promoter luciferase reporter. Transfections were performed in triplicate and were normalized to internal CMV-CAT controls. Synthetic ligand-driven reporter activity is expressed as a percentage of transfected constitutive K5-driven promoter activity. (c) The activity of the human stromelysin promoter was compared with a synthetic ligand responsive GAL4 site-driven promoter in transiently transfected primary human fibroblasts in the absence or presence of $100 \mathrm{nM}$ FK1012. In this experiment the chloramphenicol acetyl transferase reporter gene was used. Fibroblasts were transfected with synthetic ligand transcription expression and reporter plasmids or with the stromelysin-promoter reporter plasmid. Transfections were performed in triplicate and were normalized to the cotransfected constitutive cytomegalovirus (CMV)-luciferase plasmid. Reporter activity is expressed as a percentage of transfected stromelysin promoter activity. 

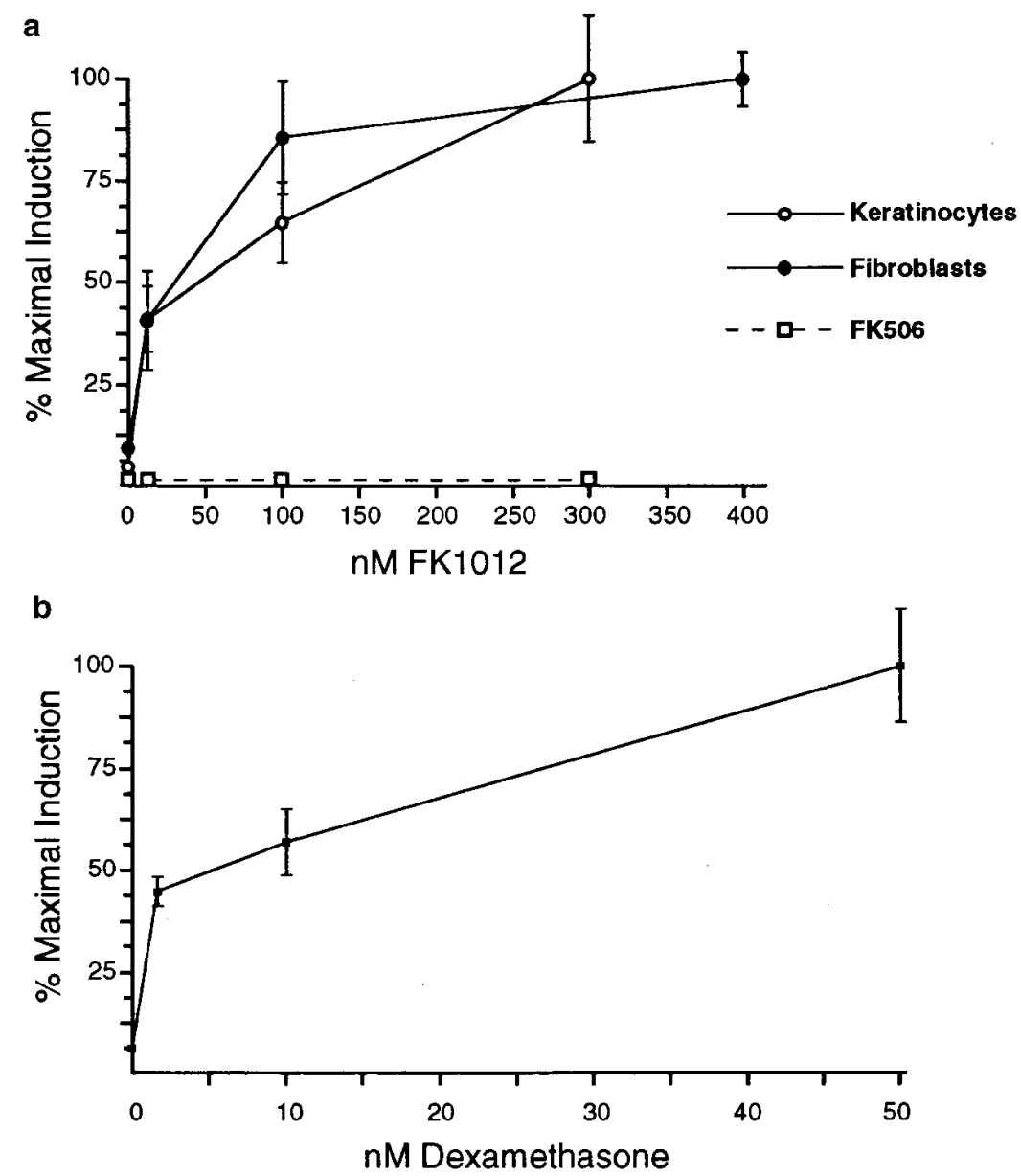

C

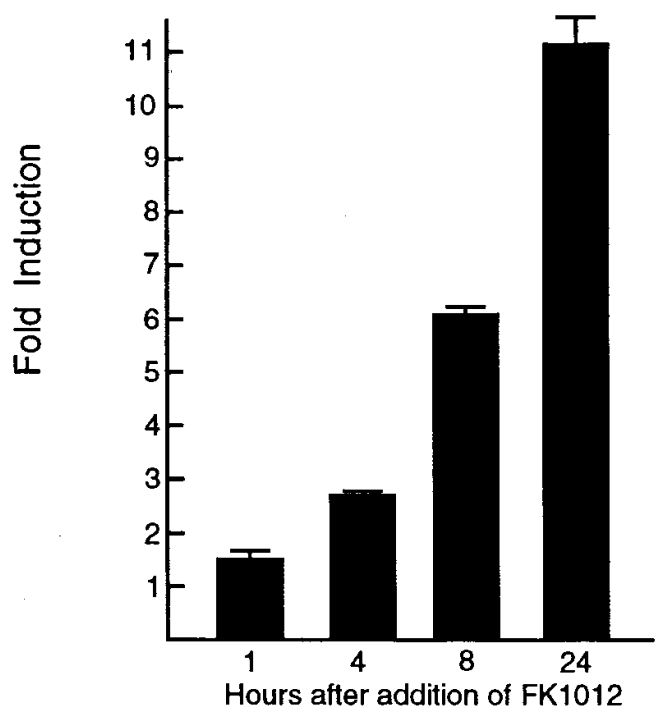

Figure 2. Concentration-dependent synthetic ligand-driven gene expression. (a) Primary human keratinocytes (open circles) and primary human fibroblasts (solid circles) were transiently transfected with synthetic ligand transcription expression and reporter plasmids. $16 \mathrm{~h}$ after gene transfer, cells were incubated with synthetic ligand in concentrations shown on the abscissa for $48 \mathrm{~h}$. The experiments were performed in triplicate independent transfections and internally controlled for transfection efficiency using the cotransfected CMV-CAT expression plasmid. FK506-M (open squares) is a modified FK506 monomer without immunosuppressive qualities that retains high affinity for FKBP. (b) As a comparison of gene induction efficacy with steroid receptor family transcription factors triggered by steroid ligand, primary human keratinocytes were cotransfected with reporter and expression plasmids for glucocorticoid-driven gene expression. $16 \mathrm{~h}$ after transfection, cells were incubated in media containing the range of dexamethasone concentrations shown on the abscissa; the experiments were performed in triplicate independent transfections, and were internally controlled for transfection efficiency using the cotransfected CMV-CAT expression plasmid. (c) Timecourse of synthetic ligand-activated target gene induction. Primary human keratinocytes were transiently transfected with synthetic ligand transcription expression and reporter plasmids. $16 \mathrm{~h}$ later media was adjusted to contain $100 \mathrm{nM}$ FK1012. Cell extracts were prepared at $0,1,4,8$, and $24 \mathrm{~h}$ after FK1012 addition and assayed for reporter gene activity. Data were normalized for transfection efficiency using CMV-CAT internal controls and are expressed as fold induction over time zero.

control the magnitude of expression of specific target genes in intact cells and tissues holds the promise of performing complex genetic experiments with quantitative biochemical precision and for dosed genetic therapies. Precise control of this magnitude in gene therapy efforts is, in a subset of cases, an absolute requirement. Our current lack of a generally effective way to accomplish this has hampered even initial efforts at correction of certain gene defects, such as in the case of mucopolysaccharidosis type I (MPS I). An attempt to correct MPS I patient fibroblasts lacking $\alpha$-L-iduronidase by transfection with the wild-type gene led to uncontrolled expression of excessive amounts of $\alpha$-L-iduronidase and the unintended devel- 


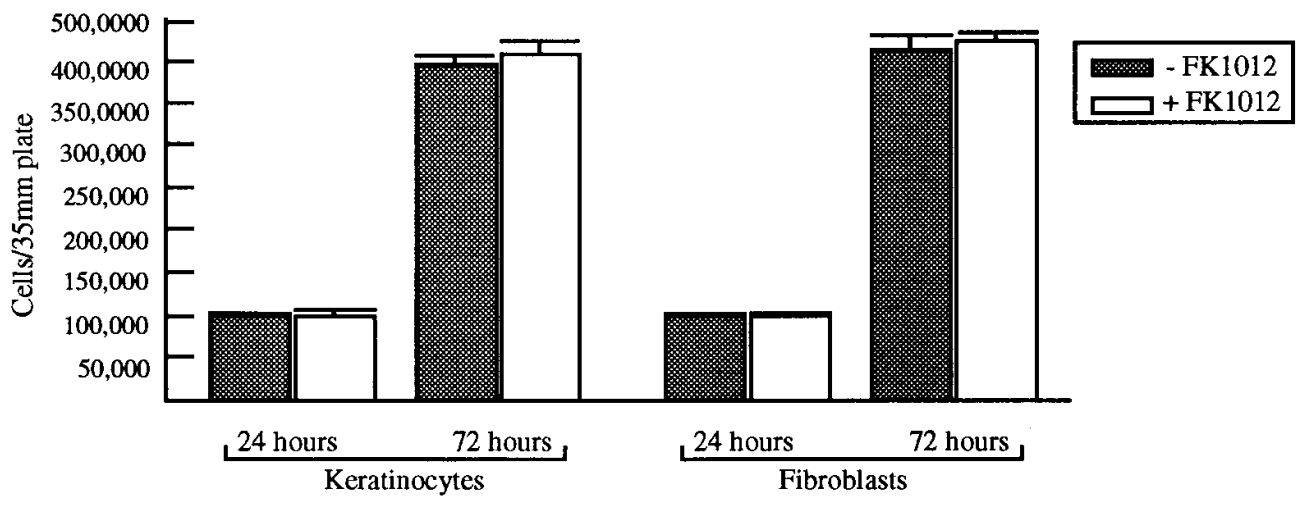

Figure 3. FK1012 does not alter proliferation kinetics of primary human keratinocytes and fibroblasts. Primary human keratinocytes and fibroblasts were seeded in 35-mm plates and grown in the presence or absence of $100 \mathrm{nM}$ FK1012 from sparse density to confluence. FK1012, in concentrations sufficient to generate induction of target gene expression, had no adverse effect on mitotic growth kinetics of primary human keratinocytes in vitro in cells growing from low density on tissue culture plates to near confluence. Primary human fibroblasts were seeded in 35-mm plates and grown in the presence or absence of 100 nM FK1012 from sparse density to confluence. Experiments were performed in triplicate.

opment of a cellular phenotype analogous to another MPS disease in the transfected cells (23). The development of general approaches to precisely regulate target gene expression, then, will represent a major advance in experimental and therapeutic biomedicine. Keratinocytes and fibroblasts can be readily removed from the skin, engineered with desired genetic elements, and grafted back to the original donor. Such engineered cells have been used to deliver a number of polypeptides to the systemic circulation, including apolipoprotein E, growth hormone, and factor IX (24-27). Topically regulated cutaneous production of therapeutic polypeptides for systemic delivery can be envisioned for a number of disorders, including production of colony stimulating factors in patients recovering from bone marrow suppression and interferon production in chronic hepatitis. Because the skin is accessible for both delivery of genetic elements as well as for topical regulation of their expression, we have focused on target gene regulation in normal keratinocytes and fibroblasts in vitro as a first step toward precise and nontoxic target gene regulation in vivo. Here we use synthetic ligand-driven targeted intranuclear oligomerization to regulate target gene expression in primary human keratinocytes and fibroblasts. The current approach is a prototype system that offers versatility in the diversity of modular DNA binding domains that can be used. Combined with development of new ligands, such an approach may ultimately allow the magnitude of many target genes to be regulated with precision simultaneously within the same cell or tissue to achieve specific biological effects. Application of this new approach to a

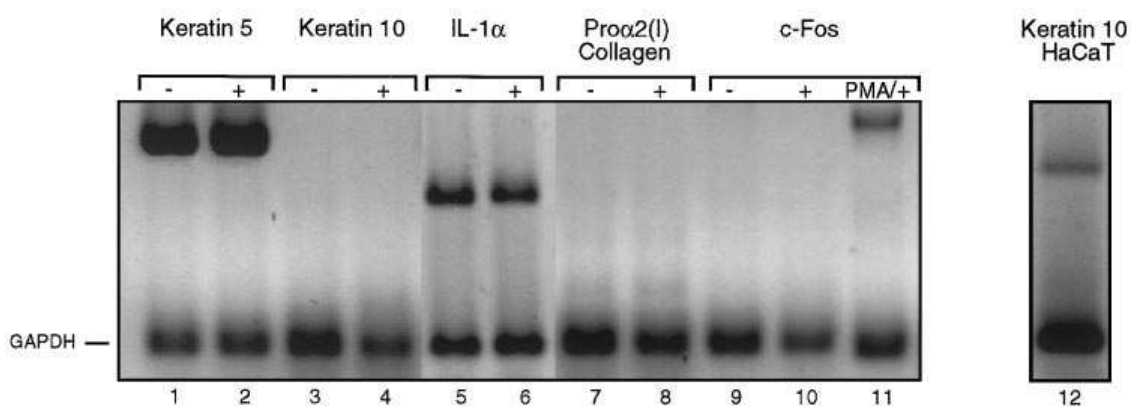

b

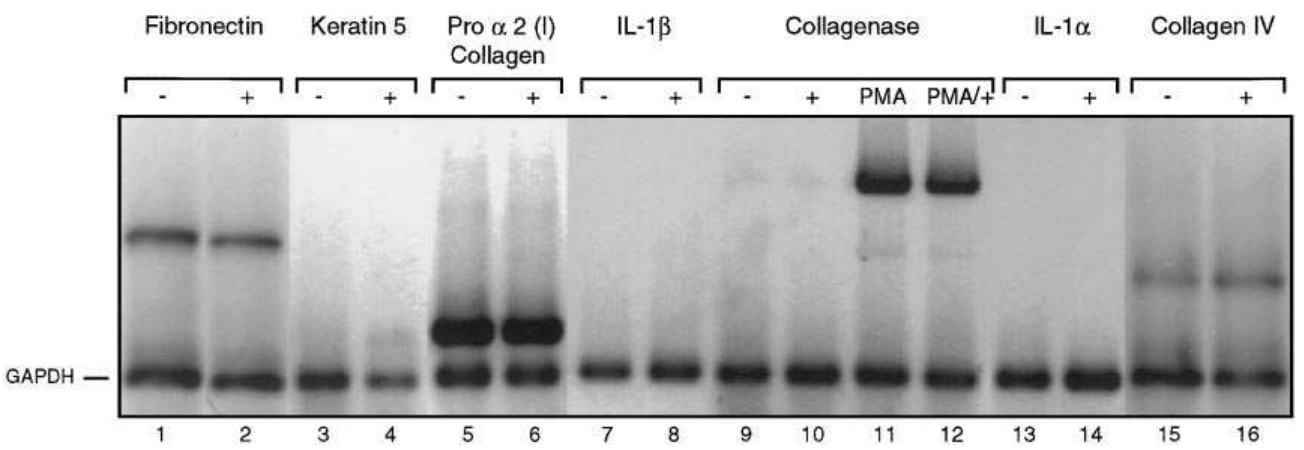

Figure 4. FK1012 does not significantly alter mRNA expression in cells of normal human skin. (a) Differentiation and tissue-specific genes in normal keratinocytes. mRNA levels of a number of constitutive and inducible genes were examined in keratinocytes in vitro in the presence or absence of FK1012. Shown are results of internally controlled reverse transcriptase PCR analysis of marker genes obtained in normal human keratinocytes grown in the presence $(+)$ or absence $(-)$ of $100 \mathrm{nM}$ FK1012. Lane 12 showing K10 expression in $\mathrm{HaCaT}$ cells is included as a positive control demonstrating detection of K10 messages in cells known to express this gene in vitro. (b) Tissue-specific genes in normal fibroblasts. Levels of mRNA expression of constitutive and inducible fibroblast-expressed genes were analyzed by internally controlled reverse transcriptase PCR analysis after growth in the presence $(+)$ or absence $(-)$ of FK1012. 
regulating the magnitude of gene expression by an apparently biologically inert intracellular dimerizing agent in primary human keratinocytes and fibroblasts reported here may find future utility in experimental and therapeutic gene regulation in the skin.

\section{Acknowledgments}

We thank G. Crabtree, D. Spencer, and S. Schreiber as well as N. Griffiths for expert administrative support.

This work was supported by the Office of Research and Development, Department of Veterans Affairs (V.A.), and the National Institutes of Health (AR4337101 and AR43799 to P.A. Khavari).

\section{References}

1. Oltvai Z.N., C.L. Milliman, and S.J. Korsmeyer. 1993. Bcl-2 heterodimerizes in vivo with a conserved homolog, Bax, that accelerates programmed cell death. Cell. 74:609-619.

2. Fenjves, E.S., J. Smith, S. Zaradic, and L.B. Taichman. 1994. Systemic delivery of secreted protein by grafts of epidermal keratinocytes: prospects for keratinocyte gene therapy. Hum. Gene Ther. 5(10):1241-1248.

3. Perez, J.R., S. Shull, G.J. Gendimenico, R.J. Capetola, J.A. Mezick, and K.R. Cutroneo. 1992. Glucocorticoid and retinoid regulation of alpha-2 type I procollagen promoter activity. J. Cell Biochem. 50:26-34.

4. Qu, Z., S. Fujimoto, and T. Taniguchi. 1994. Enhancement of interferongamma-induced major histocompatibility complex class II gene expression by expressing an antisense RNA of poly(ADP-ribose) synthetase. J. Biol. Chem. 269:5543-5547.

5. Gossen, M., S. Freundlieb, G. Bender, G. Muller, W. Hillen, and H. Bujard. 1995. Transcriptional activation by tetracyclines in mammalian cells. Science (Wash. DC). 268:1766-1769.

6. Heldin, C.H. 1995. Dimerization of cell surface receptors in signal transduction. Cell. 80:213-223.

7. Schule, R., and R.M. Evans. 1991. Cross-coupling of signal transduction pathways: zinc finger meets leucine zipper. Trends Genet. 7:377-381.

8. Davis, M.M., and P.J. Bjorkman. 1988. T-cell antigen receptor genes and T-cell recognition. Nature (Lond.). 334:395-402. Erratum appears in Nature (Lond.). 1988; 335:744.

9. Kerppola, T.K., and T. Curran. 1991. Fos-Jun heterodimers and Jun homodimers bend DNA in opposite orientations: implications for transcription factor cooperativity. Cell. 66:317-326.

10. Kolanus, W., C. Romeo, and B. Seed. 1993. T cell activation by clustered tyrosine kinases. Cell. 74:171-183.

11. Spencer, D.M., T.J. Wandless, S.L. Schreiber, and G.R. Crabtree. 1993. Controlling signal transduction with synthetic ligands [see comments]. Science (Wash. DC). 262:1019-1024.
12. Jiang, H., S. Yamamoto, and R. Kato. 1995. Induction of anagen in telogen mouse skin by topical application of FK506, a potent immunosuppressant. J. Invest. Dermatol. 104:523-525.

13. Lauerma, A.I., B.D. Stein, B. Homey, C.H. Lee, E. Bloom, and H.I Maibach. 1994. Topical FK506: suppression of allergic and irritant contact dermatitis in the guinea pig. Arch. Dermatol. Res. 286:337-340.

14. Rheinwald, J.G., and H. Green. 1975. Serial cultivation of strains of human epidermal keratinocytes: the formation of keratinizing colonies from single cells. Cell. 6:331-343.

15. Balcar, V.J., J. Shen, S. Bao, and N.J. King. 1994. Na(+)-dependent high affinity uptake of L-glutamate in primary cultures of human fibroblasts isolated from three different types of tissue. FEBS Lett. 339:50-54.

16. Jiang, C.K., D. Connolly, and M. Blumenberg. 1991. Comparison of methods for transfection of human epidermal keratinocytes. J. Invest. Dermatol. 97:969-973.

17. Ho, S.N., S.R. Biggar, D.M. Spencer, S.L. Schreiber, and G.R. Crabtree. 1996. Dimeric ligands define a role for transcriptional activation domains in reinitiation. Nature (Lond.). 382:822-826.

18. Chang, H.L., M.H. Zaroukian, and W.J. Esselman. 1989. T200 alternate exon use in murine lymphoid cells determined by reverse transcription-polymerase chain reaction. J. Immunol. 143:315-321.

19. Jiang, C.K., H.S. Epstein, M. Tomic, I.M. Freedberg, and M. Blumenberg. 1991. Functional comparison of the upstream regulatory DNA sequences of four human epidermal keratin genes. J. Invest. Dermatol. 96:162-167.

20. Unemori, E.N., C. Mauch, W. Hoeffler, Y. Kim, E.P. Amento, and E.A. Bauer. 1994. Constitutive activation of the collagenase promoter in recessive dystrophic epidermolysis bullosa fibroblasts: role of endogenously activated AP-1. Exp. Cell Res. 211:212-218.

21. Starr, D.B., W. Matsui, J.R. Thomas, and K.R. Yamamoto. 1996. Intracellular receptors use a common mechanism to interpret signaling information at response elements. Genes Dev. 10:1271-1283.

22. Breitkreutz, D., H.J. Stark, P. Plein, M. Baur, and N.E. Fusenig. 1993. Differential modulation of epidermal keratinization in immortalized ( $\mathrm{HaCaT})$ and tumorigenic human skin keratinocytes (HaCaT-ras) by retinoic acid and extracellular Ca2+. Differentiation. 54:201-217.

23. Anson, D.S., J. Bielicki, and J.J. Hopwood. 1992. Correction of mucopolysaccharidosis type I fibroblasts by retroviral-mediated transfer of the human alpha-L-iduronidase gene. Hum. Gene Ther. 3:371-379.

24. Fenjves, E.S., D.A. Gordon, L.K. Pershing, D.L. Williams, and L.B Taichman. 1989. Synthesis distribution of apolipoprotein E secreted by grafts of epidermal keratinocytes: implications for epidermal function and gene therapy. Proc. Natl. Acad. Sci. U.S.A. 86:8803-8807.

25. Gerrard, A.J., D.L. Hudson, G.G. Brownlee, and F.M. Watt. 1993. Towards gene therapy for haemophilia B using primary human keratinocytes. Nat. Genet. 3:180-183.

26. Jensen, U.B., T.G. Jensen, P.K. Jensen, J. Rygaard, B.S. Hansen, J. Fogh, S. Kolvraa, and L. Bolund. 1994. Gene transfer into cultured human epidermis and its transplantation onto immunodeficient mice: an experimental model for somatic gene therapy. J. Invest. Dermatol. 103:391-394.

27. Krueger, G.G., J.R. Morgan, C.M. Jorgensen, L. Schmidt, H.L. Li, M.K. Kwan, S.T. Boyce, H.S. Wiley, J. Kaplan, and M.J. Petersen. 1994. Genetically modified skin to treat disease: potential and limitations. J. Invest. Dermatol. 103:76S-84S. 